\title{
Minimizing early relapse and maximizing treatment outcomes in hormone-sensitive postmenopausal breast cancer: efficacy review of AI trials
}

\author{
Christos J. Markopoulos
}

Published online: 10 September 2010

(C) The Author(s) 2010. This article is published with open access at Springerlink.com

\begin{abstract}
Breast cancer is one of the leading causes of cancer-related deaths in women. Regardless of prognosis, all women with breast cancer are at risk for early recurrence. Nearly $50 \%$ of early recurrences occur within 5 years of surgery, and they peak at 2 years after surgery in women treated with adjuvant tamoxifen. Most early recurrences are distant metastases, which strongly correlate with increased mortality. Treatments that mitigate the risk of early distant metastases (DM) are, therefore, likely to improve overall survival in women with early breast cancer (EBC). Aromatase inhibitors (AIs) — anastrozole, letrozole, and exemestane- have been investigated as alternatives to tamoxifen for adjuvant treatment of hormone receptorpositive $(\mathrm{HR}+) \mathrm{EBC}$ in postmenopausal women (PMW). AIs are better at minimizing risk of early relapse compared with tamoxifen. However, it is not clear if preferential use of AIs over tamoxifen will benefit all PMW with HR+ EBC. The ability to subtype HR+ breast cancer on the basis of biomarkers predictive of response to AIs and tamoxifen would likely be key to determining the most beneficial hormonal treatment within patient subpopulations, but this process requires thorough investigation. Until then, adjuvant therapies that provide the greatest reduction in risk of DM should be considered for all PMW with HR+ EBC. This article reviews the clinical trials of AI adjuvant therapies for hormone-sensitive breast cancer, particularly in the context of how they compare with tamoxifen in
\end{abstract}

C. J. Markopoulos $(\bowtie)$

Athens University Medical School,

8, Iassiou str,

11521 Athens, Greece

e-mail: cmarkop@hol.gr

C.J. Markopoulos

e-mail: cmbreast@yahoo.gr minimizing the risk of relapse, occurrence of DM, and breast cancer-related deaths.

Keywords Aromatase inhibitors · Distant metastasis . Anastrozole $\cdot$ Letrozole $\cdot$ Exemestane $\cdot$ Tamoxifen

\section{Introduction}

Breast cancer is the most frequently diagnosed cancer in women and the second leading cause of cancer-related death in women. In the USA alone, an estimated 192,370 new cases of invasive breast cancer with roughly 40,170 breast cancer deaths were expected to occur in 2009 [1]. Over two thirds of breast cancers are hormone-sensitive and thus candidates for endocrine therapy. Endocrine therapy removes estrogen from the body or inhibits estrogen from binding to cancer cells and is the principal adjuvant treatment given to postmenopausal women (PMW) with hormone receptor-positive (HR+) early breast cancer (EBC) in an attempt to minimize the risk of relapse. The risk of relapse, which is greatest during the first 5 years of surgery, peaks at approximately 2 years [2-4]. At this peak, distant metastases (DM) are the most common type of recurrence, compared with locoregional or contralateral recurrences $[4,5]$.

DM comprise between $50 \%$ and $75 \%$ of all early recurrences $[3,5,6]$, and their appearance is the harbinger of breast cancer-related death. The 5-year survival rate for patients with DM is much lower than for patients with locoregional, contralateral, and no recurrence [5]. Therefore, reducing the risk of DM is an important therapeutic goal [5, 7-10]. Adjuvant endocrine therapies that are most effective in reducing the risk of DM may have the most favorable impact on survival in PMW with EBC. For over 
two decades, tamoxifen was thought to be the optimal adjuvant therapy for PMW with HR+ EBC. Recent studies have challenged that notion, leading to the consideration of the aromatase inhibitors (AIs) anastrozole, letrozole, and exemestane as alternatives to tamoxifen [11-16].

Until recently, the use of upfront monotherapy with an AI (i.e., instead of tamoxifen) versus a sequential/switch strategy (i.e., after some period of initial tamoxifen) was a matter of considerable debate. However, the importance of upfront AI therapy is now widely recognized, particularly in patients at higher risk of early relapse [17]. Indeed, recent data from the Breast International Group (BIG) 1-98 trial comparing a sequential strategy with tamoxifen followed by an AI (letrozole) with AI monotherapy has demonstrated that sequencing is not superior to monotherapy with an AI (letrozole) alone [16]. Major clinical trials of adjuvant therapies for hormone-sensitive breast cancer, which compare the efficacy and safety of AIs with that of tamoxifen in minimizing the risk of recurrence, DM, and breast cancer-related deaths, are discussed below.

\section{Risk of early relapse and distant metastasis}

All patients with breast cancer are at risk for early relapse. The risk of recurrence is greatest during the first few years following surgery [2-4, 6]. In a recent retrospective analysis of 3,614 PMW with estrogen receptor-positive $(\mathrm{ER}+) \mathrm{EBC}$ who were treated with tamoxifen following surgery, the annual recurrence rate overall was $4.3 \%$, and most of these (3.4\%) were DM [4] (Fig. 1). Locoregional and contralateral recurrences did not exceed $1 \%$ at any time during the study. During the 5-year median follow-up of this patient cohort, 476 developed recurrences, and of these, 344 were DM. Patients with DM had the poorest outcomes: $50 \%$ died within 0.65 years $(7.8$ months $)$ from the time of DM and 75.6\% (260/344) died overall [8]. Lamerato et al. have consistently reported DM to be the most common site of recurrence $(58.3 \%)$, with only $26.1 \%$ of recurrences

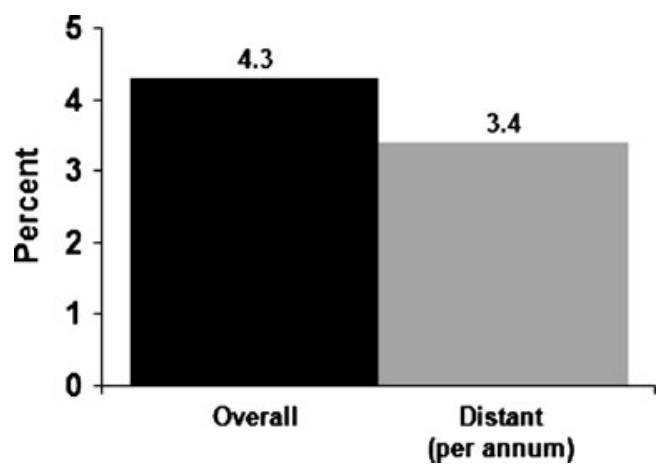

Fig. 1 Prevalence of distant metastases at 2-year peak of overall recurrences [4] locoregional, and $15.6 \%$ contralateral [5]. The 5-year probability of overall survival (OS) was poorest for women with DM (41.3\%) compared with those with locoregional $(59.3 \%)$, contralateral $(83.4 \%)$, or no recurrence $(91.7 \%)$ [5].

Factors predictive of early recurrence include hormone receptor (ER and/or progesterone receptor $(\mathrm{PgR}))$ status, degree of nodal involvement, and tumor size and tumor grade [4, 6, 18]. Patients who have tumors involving $>3$ nodes, tumors $>5 \mathrm{~cm}$, or grade III tumors and who are receiving adjuvant tamoxifen have about a three-fold increase in risk of recurrence (all $P<0.001$ ) [4]. However, individual risk factors such as node-negative $(\mathrm{N}-)$ status must be considered along with other factors. For example, according to the St. Gallen risk classification, patients with $\mathrm{N}$ - tumors that are large $(>2 \mathrm{~cm}$ ), high-grade (histologic and/or nuclear grades 2 and 3), or show evidence of peritumoral vascular invasion are considered intermediate risk [19]. It should be noted, however, that while these factors help assess the overall risk for early recurrence, they are not taken into account when determining the treatment strategy, as almost all tumors that show evidence of endocrine responsiveness (i.e., the presence of any ER) are considered candidates for endocrine therapy [17].

Recent evidence suggests that Ki67 labeling index (LI), a measure of tumor proliferative capacity, could identify patients at increased risk for poor outcomes within the St. Gallen risk classification and Adjuvant! Online risk categories [20]. Specifically, those with high Ki67 LI $(\geq 10 \%)$ had significantly poorer disease-free survival (DFS) in both the intermediate $(91.9 \%$ vs. $86.3 \% ; P=$ $0.01)$ and high-risk groups $(82.5 \%$ vs. $61.4 \% ; P=0.01)$ of St. Gallen 2007, compared with those with low Ki67 LI [20].

The potential utility of gene expression profiles [21] in the accurate prediction of risk recurrence and treatment response is also being explored. The prognostic and predictive value of the Oncotype DX recurrence score assay using the 21-gene tumor signature has been validated in $\mathrm{ER}+, \mathrm{N}-$ patients receiving tamoxifen [22, 23]. The phase 3 Trial Assigning Individualized Options for Treatment was designed to determine the best individualized treatment for $\mathrm{PMW}$ with $\mathrm{ER}+, \mathrm{N}-$ breast cancer, with a recurrence score of 11-25 [24]. The value of the Oncotype DX recurrence score has been recently investigated in major clinical trials of AIs. Recent results of the Trans Arimidex, Tamoxifen Alone or in Combination (ATAC) trial indicate that Oncotype DX is an independent predictor of risk of $\mathrm{DM}$ in $\mathrm{N}-$ and $\mathrm{N}+, \mathrm{HR}+$ patients; however, the data were not predictive of a differential benefit between anastrozole and tamoxifen [25]. A prospectively controlled study of 6,000 women with $\mathrm{N}$ - breast cancer (microarray in node-negative and one to three positive-lymph node-disease 
may avoid chemotherapy) compares a 70-gene expression signature (Mammaprint) with common clinical-pathological criteria to select patients for adjuvant chemotherapy [26].

Thus, although several clinical and molecular prognostic factors for early risk of DM are known, the ability to accurately predict a favorable response to a particular adjuvant therapy remains elusive. Advanced methods of individual risk assessment must be rigorously validated before treatment strategies can be tailored to a patient's unique molecular profile. Until then, all PMW with HR+ EBC should be considered at risk for recurrence and candidates for adjuvant endocrine therapy, to minimize the risk of DM and improve the chances of survival [17].

\section{Adjuvant tamoxifen therapy and impact on DM}

Until recently, on the basis of previous clinical trial results [27], 5 years of tamoxifen was the standard adjuvant treatment for PMW with HR+ EBC. Meta-analysis of the Early Breast Cancer Trialists' Collaborative Group (EBCTCG) showed that, at a median follow-up of 15 years, 5 years of adjuvant tamoxifen therapy significantly reduced recurrence risk by $41 \%$ and mortality by $34 \%$ in patients with ER+ tumors compared with placebo. Adjuvant tamoxifen therapy reduced the risk of local recurrences by $53 \%$ (hazard ratio $(\mathrm{HR})=0.47 ; P<0.00001$ ) and the risk of DM by $36 \%(\mathrm{HR}=0.64 ; P<0.00001) \quad[27,28]$. Despite these findings, many patients receiving tamoxifen continue to experience recurrences, $50 \%$ of which occur within the first 2 to 3 years after surgery, a period when DMs comprise between $50 \%$ and $75 \%$ of recurrences $[3,4,6]$.

Trials such as the National Surgical Adjuvant Breast and Bowel Project (NSABP) B-14 study and the Stockholm Tamoxifen trial indicate that tamoxifen therapy reduces the risk of recurrence, but primarily for locoregional recurrences and not the more serious and life-threatening DM [29, 30]. Also, recent studies have demonstrated that PMW with $\mathrm{ER}+\mathrm{EBC}$ who are homozygous for variant alleles of cytochrome P (CYP) 2D6 are unable to metabolize tamoxifen effectively [31,32]. Consistently, women receiving tamoxifen in combination with a CYP2D6 inhibitor display an increased risk for breast cancer recurrence compared with those receiving tamoxifen alone (2-year recurrence $13.9 \%$ vs. $7.5 \%$; $\mathrm{HR}=1.92 ; P<0.001$ ) [33]. These findings led the Food and Drug Administration to recommend relabeling tamoxifen to reflect the possible negative effects on outcomes in women may have reduced CYP2D6 activity. In addition, because of its estrogenagonistic activity in selected tissues, tamoxifen is associated with serious adverse events (SAEs) such as endometrial cancer and thromboembolic events [34].

\section{AIs and treatment strategy trials}

Third-generation AIs include anastrozole, letrozole, and exemestane. Anastrozole and letrozole are nonsteroidal drugs that block estrogen synthesis through a competitive inhibition of aromatase, while exemestane is a steroidal AI that blocks estrogen synthesis by irreversibly binding with aromatase and inhibiting its activity [35]. All three AIs almost completely block estrogen synthesis and have been approved by the Food and Drug Administration for the treatment of early-stage breast cancer because of their efficacy and tolerability in a variety of clinical settings [36]. However, there is evidence that letrozole provides a greater suppression of both plasma (residual estradiol (after 6 weeks of AI therapy): $4.8 \%$ letrozole vs. $7.2 \%$ anastrozole, $P=$ 0.018 ; residual estrone: $1.2 \%$ letrozole vs. $3.7 \%$ anastrozole, $P=0.003$; residual estrone sulfate: $1.1 \%$ letrozole vs. $4.7 \%$ anastrozole, $P=0.003$ ) and tissue (residual estradiol (after 16 weeks of AI therapy): $2.4 \%$ letrozole vs. $11.0 \%$ anastrozole, $P=$ not reported (NR); residual estrone: $9.3 \%$ letrozole vs. $16.6 \%$ anastrozole, $P=\mathrm{NR}$; residual estrone sulfate: $9.9 \%$ letrozole vs. $27.1 \%$ anastrozole, $P=\mathrm{NR}$ ) estrogen levels compared with anastrozole [37]. Clinical trials of adjuvant AIs in PMW with $\mathrm{HR}+$ breast cancer include initial adjuvant, extended adjuvant, switch, and sequential trials (Fig. 2).

Upfront adjuvant trials - the ATAC trial, BIG 1-98 (monotherapy arms), and tamoxifen exemestane adjuvant multicenter (TEAM) trials - randomized newly diagnosed patients immediately following surgery and administered 5 years of treatment with either tamoxifen, an AI, or a sequence of tamoxifen and $\mathrm{AI}[11,12,15,16,38,39]$. The TEAM trial was designed to compare tamoxifen with the

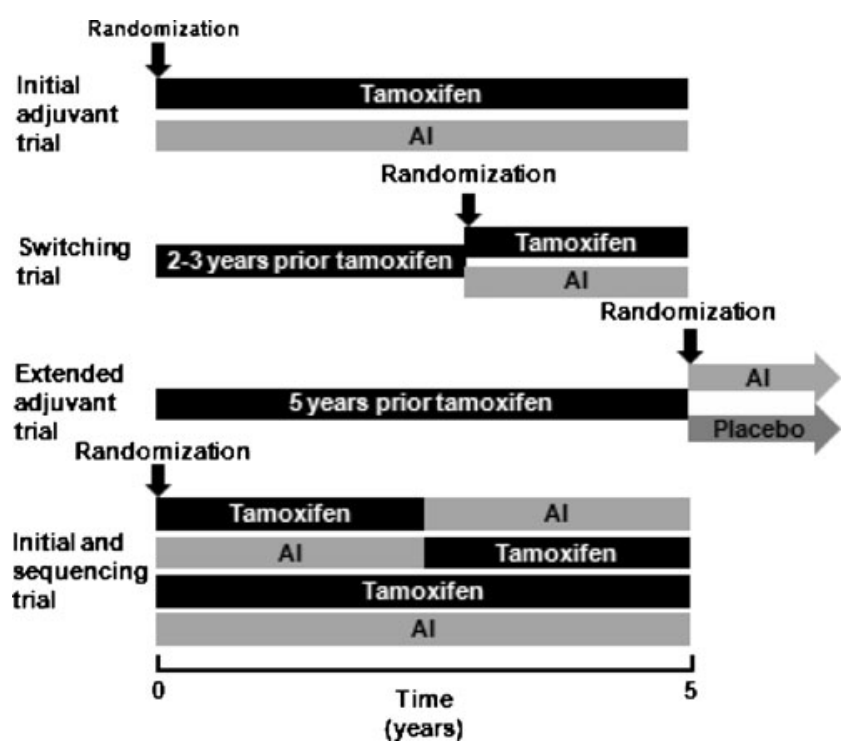

Fig. 2 Types of adjuvant trials. $A I$ aromatase inhibitor 
AI exemestane only at 2.75 years (first primary endpoint) [39]; the trial was amended to compare exemestane with sequential use of tamoxifen to exemestane at 5 years (second primary endpoint) [40].

Switch adjuvant AI trials, such as the Intergroup Exemestane Study (IES), Italian Tamoxifen Anastrozole (ITA) trial, the Austrian Breast and Colorectal Study Group 8 (ABCSG 8), and ARimidex NOlvadex (ARNO) 95 trials randomized disease-free patients who received 2 to 3 years of adjuvant therapy with tamoxifen as part of a 5-year course and compared the relative efficacy of continuing their treatment with tamoxifen or switching to an AI [14, 41-43]. Only patients who are disease-free at the time of randomization are allowed to continue in switch trials, excluding patients whose disease recurs during the first 2 to 3 years of tamoxifen therapy. This is different from sequential adjuvant trials (BIG 1-98 sequential arms and TEAM), which include all recurrences after randomization in their efficacy analysis, including events during the early peak at 2 to 3 years $[2,4]$. Thus, while sequential trials are designed to assess the benefit of sequential therapy versus monotherapy in newly diagnosed patients, switch trials primarily address whether the risk of an event beyond 2 years is reduced by switching to an AI.

Lastly, extended adjuvant trials, such as the National Cancer Institute of Canada MA.17 Trial, ABCSG 6, and the NSABP B-33 trial, are designed to evaluate the benefit of additional endocrine therapy by randomizing patients who have already received a standard 5-year course of tamoxifen to further treatment with an $\mathrm{AI}$ or placebo (or no treatment in the case of the open-label ABCSG-6A trial) [44-47]. The different strategies that have been examined (Fig. 2) across multiple AI trials thus provide a number of treatment options for endocrine therapy in PMW with EBC.

\section{Upfront AI therapy and impact on DM}

\subsection{ATAC: upfront therapy with anastrozole}

This was a large, double-blind, randomized, non-inferiority/ superiority comparison of anastrozole $(n=3,125)$ with tamoxifen $(n=3,116)$ or a combination of anastrozole and tamoxifen $(n=3,125)$ for 5 years in PMW with EBC. The combination arm was discontinued after the initial analysis for lack of efficacy; thus, a third of the trial participants were lost [12, 38]. Importantly, because the trial was designed and implemented soon after the initial introduction of AIs as adjuvant therapy, the population was not restricted to patients with $\mathrm{HR}+$ disease [38] but also included patients with HR-unknown tumors $(16.3 \%$ of the total trial population). Subsequent analyses have thus focused on the comparison of anastrozole with tamoxifen in both the intent-to-treat (ITT) population and the more relevant subgroup of $\mathrm{HR}+$ patients $(n=5,216$ of the original $9,366)[12,15]$. At a median follow-up of 33.3 months, anastrozole demonstrated significantly better DFS compared with tamoxifen $(\mathrm{HR}=0.87 ; P=0.005)$ and with the combination of anastrozole and tamoxifen $(\mathrm{HR}=0.76$; $P=0.002$ ). Time to recurrence (TTR) was significantly longer in the anastrozole group than in the tamoxifen group (HR $=0.73, P=0.003$ ), but there was no significant difference between tamoxifen and the combination groups (HR $=$ $1.09 ; P=0.4)$. These initial results do not provide information on DM rates and survival, because this was an eventdriven analysis requiring at least 704 distant recurrences, and only 615 were recorded [38].

At a median of 68 months of follow-up, DFS was significantly better with anastrozole versus tamoxifen in $\mathrm{HR}+$ patients $(\mathrm{HR}=0.83 ; P=0.005)$, as was TTR (HR=0.74; $P=0.0002)$, but time to distant recurrence (TTDR) was not significantly different between anastrozole- and tamoxifen-treated groups in the $\mathrm{HR}+$ population ( $\mathrm{HR}=0.84 ; P=0.06$; Table 1$)$ [12]. Findings at the 100-month follow-up also demonstrated that anastrozole was superior to tamoxifen in improving DFS ( $\mathrm{HR}=$ $0.85 ; P=0.003)$ and TTR $(\mathrm{HR}=0.76 ; P=0.0001)$, with a particular benefit in locoregional and contralateral breast cancer recurrence. At this follow-up post treatment, there were $16 \%$ fewer $\mathrm{DM}$ in the $\mathrm{HR}+$ population $(\mathrm{HR}=0.84 ; P=$ 0.022; 305 anastrozole vs. 357 tamoxifen events) [15]. However, it is important to note that significant improvement in TTDR was achieved post-treatment only after a median follow-up of 100 months [15].

Disappointingly, while anastrozole appeared to provide significant benefit with the improvement in DFS, no improvement was seen in survival with more than 9 years of follow-up. OS ( $\mathrm{HR}=0.97 ; P=0.7 ; 472$ anastrozole vs. 477 tamoxifen deaths), deaths after recurrence ( $\mathrm{HR}=0.90$; $P=0.2 ; 245$ anastrozole vs. 269 tamoxifen deaths), and deaths without recurrence $(\mathrm{HR}=1.05 ; P=0.6 ; 227$ anastrozole vs. 208 tamoxifen deaths) were not significantly different between the two groups [15]. A retrospective analysis of ATAC at 2.5 years, undertaken to better understand the types of early recurrences in the ITT population, indicated fewer locoregional, DM, and contralateral recurrences with anastrozole compared with tamoxifen, but the greatest benefit with anastrozole was in reducing the risk of locoregional and contralateral events with less impact on DM (7\% reduction; Fig. 3) [3]. A recently reported retrospective analysis of events at 2 years in the HR+ population of ATAC also reported fewer overall recurrences with anastrozole relative to tamoxifen, but the reduction in $\mathrm{DM}$ was not significant $(\mathrm{HR}=0.79 ; 95 \% \mathrm{CI}$, 0.58-1.07) [48]. The AEs profile of ATAC displayed a greater incidence of fractures (11\% anastrozole vs. $7.7 \%$ 
Table 1 Efficacy end points from upfront adjuvant trials comparing tamoxifen and aromatase inhibitors

\begin{tabular}{lllll}
\hline Initial adjuvant trials & Endpoint & F/U (months) & HR for recurrence & $P$ value \\
\hline BIG 1-98 (letrozole) & TTDR & $25.8(\mathrm{PCA})$ & $0.73^{\mathrm{a}}$ & 0.001 \\
& & $76(\mathrm{MAA})$ & $0.85^{\mathrm{a}}$ & 0.05 \\
& DFS & $25.8(\mathrm{PCA})$ & $0.81^{\mathrm{a}}$ & 0.003 \\
& & $76(\mathrm{MAA})$ & $0.88^{\mathrm{a}}$ & 0.03 \\
& OS & $25.8(\mathrm{PCA})$ & $0.86^{\mathrm{a}}$ & 0.16 \\
ATAC (anastrozole) & TTR & 68 & $0.87^{\mathrm{a}}$ & 0.08 \\
& & 100 & $0.74^{\mathrm{b}}$ & 0.0002 \\
& TTDR & 68 & $0.76^{\mathrm{b}}$ & 0.0001 \\
& & 100 & $0.84^{\mathrm{b}}$ & 0.06 \\
& DFS & 68 & $0.84^{\mathrm{b}}$ & 0.022 \\
& & 100 & $0.83^{\mathrm{b}}$ & 0.01 \\
& OS & 68 & $0.85^{\mathrm{b}}$ & 0.003 \\
& & 100 & $0.97^{\mathrm{b}}$ & 0.7 \\
\hline
\end{tabular}

Source: $[11,12,15,16]$

$A T A C$ arimidex, tamoxifen

alone or in combination, $B I G$

Breast International Group,

$D F S$ disease-free survival, $H R$

hazard ratio, $O S$ overall

survival, $M A A$ monotherapy

arm analysis, $P C A$ primary

core analysis, TTDR

time to distant recurrence,

TTR time to recurrence

${ }^{\mathrm{a}}$ ITT population

${ }^{\mathrm{b}} \mathrm{HR}+$ population tamoxifen; $P<0.0001)$ and arthralgia $(35.6 \%$ anastrozole vs. $29.4 \%$ tamoxifen; $P<0.0001)$ in anastrozole-treated women in contrast to a greater incidence of endometrial cancer $(0.2 \%$ anastrozole vs. $0.8 \%$ tamoxifen; $P=0.02)$, venous thromboembolic events $(2.8 \%$ anastrozole vs. $4.5 \%$ tamoxifen; $P=0.0004$ ), and gynecologic abnormalities (i.e., vaginal bleeding, discharge, hot flushes; all $P<0.0001$ ) in tamoxifen-treated women [12].

\subsection{BIG 1-98: upfront therapy with letrozole}

This large, double-blind, randomized, superiority trial was designed and implemented by the IBCSG to evaluate the efficacy of letrozole versus tamoxifen monotherapy, or letrozole and tamoxifen as sequential therapy, in either order, compared with letrozole alone as initial adjuvant endocrine therapy, in PMW with EBC $(N=8,010)$. BIG 198 was comprised of a four-arm option which assigned patients to 5 years of letrozole $(n=1,546) ; 5$ years of tamoxifen $(n=1,548)$; 3 years of tamoxifen after 2 years of letrozole $(n=1,540)$; and 3 years of letrozole after 2 years of tamoxifen $(n=1,548)$, and a two-arm option, which assigned patients to 5 years of letrozole $(n=917)$ or tamoxifen $(n=911)$ therapy [11].

The BIG 1-98 trial is uniquely designed to study two key questions regarding the use of AIs and tamoxifen: 1) the efficacy of letrozole monotherapy versus tamoxifen monotherapy $[11,16,49]$ and 2) whether sequential therapy, in either order, is superior to monotherapy [16]. Unlike the ATAC trial, BIG 1-98 required patients' tumors to be $\mathrm{HR}+$, defined as positive for ER, PgR, or both [11]. The first report from BIG 1-98 $(N=8,010)$, the primary core analysis (PCA), was designed to compare letrozole and tamoxifen as initial adjuvant therapy. The PCA included events from the
Fig. 3 Absolute reduction in overall recurrences and distant metastases in the a Arimidex, Tamoxifen Alone, or Combination trial (hormone receptor-positive patients) at 2.5 years [3] and b Breast International Group $1-98$ at 2 years [6]. $A N A$ anastrozole; $L E T$ letrozole; TAM tamoxifen 
four- and two-arm option, including events from the initial treatment period of the two sequential arms up to 30 days after the switch, thus increasing the statistical power of the comparison (Fig. 4).

At a median follow-up of 25.8 months, the PCA demonstrated that letrozole treatment significantly improved DFS (HR $=0.81 ; P=0.003 ; 351$ letrozole vs. 428 tamoxifen events) and TTR $(\mathrm{HR}=0.72 ; P<0.001)$ and produced a significant $27 \%$ reduction in the risk of DM (TTDR) compared with tamoxifen ( $\mathrm{HR}=0.73 ; P=0.001 ; 184$ letrozole vs. 249 tamoxifen events). OS did not reach statistical significance at this early follow-up ( $\mathrm{HR}=0.86$; $P=0.16$; 166 letrozole vs. 192 tamoxifen events; Table 1) [11]. A retrospective analysis examining the predictors of early relapse in BIG $1-98(N=7,707)$ at 2 years found that letrozole reduced the risk of both overall recurrence and DM (87 letrozole vs. 125 tamoxifen events) by $30 \%$ (Fig. 3) [6]. This is particularly important given the preponderance of $\mathrm{DM}$ at this time point [4] and the impact of DM on survival $[5,8]$. Based on the 2005 PCA results and the superiority of letrozole over tamoxifen, the IBCSG decided to unblind the tamoxifen monotherapy arm and allow patients to cross over to the letrozole arm. The other three arms remained blinded [16].

Upon being counseled regarding the superiority of letrozole versus tamoxifen, 619 patients $(25.2 \%)$ on tamoxifen monotherapy chose to cross over to letrozole; most did so in 3-5 years and received letrozole for a median of 18 months. Subsequent analyses of the PCA (60.5 months), and the analysis of the monotherapy arms (MAA) at 76 months, were affected by this crossover of patients who were at high risk for recurrence, most likely in favor of those in the tamoxifen arm, who benefited by the early switch to letrozole. Therefore, data were presented as protocol-defined ITT population (including crossover patients) and a second analysis that censored patients at the time of crossover. At a median follow-up of 76 months, an analysis of the ITT population in the MAA demonstrated consistent benefit, with a significant improvement in DFS $(\mathrm{HR}=0.88 ; 95 \% \mathrm{CI}, 0.78-0.99 ; P=0.03)$ and TTDR $(\mathrm{HR}=$ $0.85 ; 95 \%$ CI, $0.72-1.00 ; P=0.05)$ with letrozole [16]. Letrozole also demonstrated a strong trend, despite the crossover, for improved OS (303 letrozole vs. 343 tamoxifen events; $\mathrm{HR}=0.87 ; 95 \% \mathrm{CI}, 0.75-1.02 ; P=$ $0.08)$. In the censored analysis, results demonstrated even greater benefit with letrozole. Patients receiving letrozole had significant improvements in DFS (HR $=0.84 ; 95 \% \mathrm{CI}$, $0.74-0.95)$, TTDR (HR $=0.81 ; 95 \% \mathrm{CI}, 0.68-0.96)$, and OS ( $\mathrm{HR}=0.81 ; 95 \% \mathrm{CI}, 0.69-0.94)$.

There were 40 fewer deaths in the letrozole groups compared with the tamoxifen group (303 vs. 343 ) and an identical number of non-breast cancer-related deaths in each group ( 87 vs. 87 ), indicating that the OS benefit provided by letrozole is due to improved cancer survival. Consistent with the findings of the MAA at 76 months' median followup, the 60.5 months' median follow-up of the ITT population in the PCA also found a benefit in DFS (HR= $0.86 ; 95 \%$ CI, $0.77-0.96 ; P=0.008)$, TTR (HR $=0.82 ; 95 \%$ CI, $0.71-0.95 ; P=0.004$ ), and TTDR (HR $=0.79 ; 95 \% \mathrm{CI}$, $0.68-0.92 ; P=0.003)$. There was also a trend toward improved OS with letrozole ( $\mathrm{HR}=0.87 ; 95 \% \mathrm{CI}, 0.75$ $1.01 ; P=0.07)$ [49]. In the censored analysis, patients receiving letrozole demonstrated significant benefits in DFS $(\mathrm{HR}=0.83 ; 95 \% \mathrm{CI}, 0.74-0.93)$, TTR $(\mathrm{HR}=0.79$; 95\% CI, 0.68-0.90), and TTDR (HR $=0.78 ; 95 \%$ CI, $0.67-$ 0.92). Importantly, there was a significant benefit in OS $(\mathrm{HR}=0.81 ; 95 \% \mathrm{CI}, 0.70-0.94)$ [49]. However, the IBCSG concluded that early crossover to letrozole, although ethical
Fig. 4 Breast International Group 1-98 schema/analysis. $C T$ chemotherapy; ITT intent-totreat

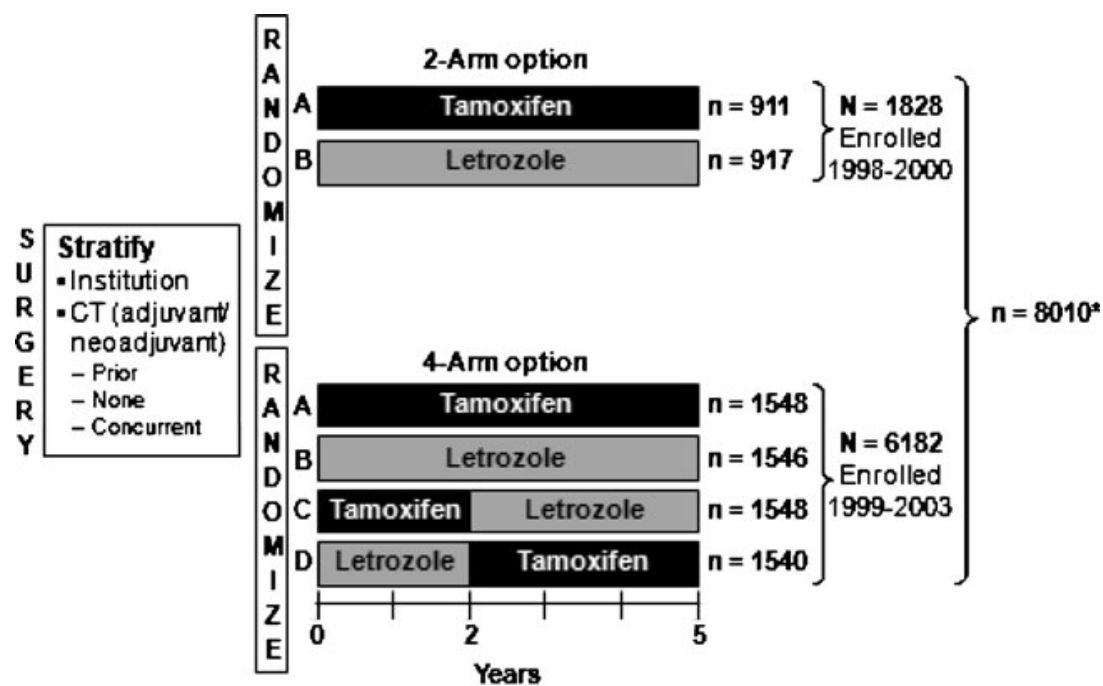

-TT. Exdudes 18 patents who withdrew consert and dd not recelve study treatment. 
and appropriate because of the significant survival benefit, likely biased the ITT analysis in favor of tamoxifen and the censored analysis in favor of letrozole, and makes accurate assessments of OS difficult. Therefore, to adjust for the potential bias due to crossover, an additional Inverse Probability of Censoring Weighted (IPCW) analysis [50] was undertaken to provide a more accurate estimate of the clinical benefit of letrozole. The results of the analysis not only underscored the importance of validated methods, such as the IPCW analysis, but also unequivocally demonstrated that 5 years of letrozole significantly improved DFS by $15 \%(\mathrm{HR}=0.85 ; 95 \% \mathrm{CI}, 0.76-0.96)$ and $\mathrm{OS}$ by $17 \%(\mathrm{HR}=0.83 ; 95 \% \mathrm{CI}, 0.7-0.97)$ [51], and reflected the statistics that might have been observed in the absence of selective crossover [16].

The 60.5-month update of the PCA population corroborates findings of the 76-month monotherapy analysis showing an emergent benefit of letrozole on survival with long-term follow-up [16]. The survival benefit with letrozole, not evident when anastrozole was compared with tamoxifen in the ATAC trial, underscores the substantial benefit realized when early DM are controlled, as illustrated by the PCA and the retrospective analysis examining the predictors of early relapse at 2 years $[6,11]$. This is the first time an upfront adjuvant trial has demonstrated improvement in OS, and it provides further evidence that preventing early DM translates into a long-term survival advantage. Findings from the safety analysis reported with the 60.5month update of the PCA population were consistent with those of the initial PCA analysis, and with known AEs of letrozole and tamoxifen, without evidence of risk increasing over time [49].

\subsection{FACE: upfront therapy-letrozole versus anastrozole}

The randomized, open-label Femara Anastrazole Clinical Evaluation (FACE) trial was designed to compare the safety and efficacy of anastrozole versus letrozole in the upfront AI treatment of PMW with HR+ and N+ breast cancer [52, 53]. The primary outcome measures are rate of DFS at 5 years between letrozole and anastrozole; secondary outcome measures include safety, efficacy, OS, and time to DM $[52,53]$. Although results from the ATAC and BIG 1-98 trials allow for indirect comparisons, forthcoming results from FACE should determine conclusively whether any important efficacy differences exist between these two nonsteroidal AIs.

\subsection{TEAM: upfront therapy with exemestane}

In this randomized, multinational, open-label study of PMW with HR+ EBC $(N=9,300)$, investigators in nine countries followed nearly identical protocols, with minor differences based on local guidelines [54]. The trial was originally designed to evaluate the efficacy and safety of 5 years of initial monotherapy with exemestane or tamoxifen. However, based on the initial results of the IES [41], the design was amended; women randomized to tamoxifen had to switch after 2.5-3 years to exemestane for the remainder of the 5 years. Therefore, the final design compared exemestane monotherapy with sequential tamoxifen to exemestane therapy for 5 years and compared exemestane monotherapy with tamoxifen monotherapy at 2.75 years. At 2.75 years, there was no statistically significant difference in DFS with tamoxifen versus exemestane monotherapy ( $\mathrm{HR}=0.89 ; P=0.12)$, although improvement in the secondary endpoint of time to DM was observed $(\mathrm{HR}=0.81 ; P<0.03)[39]$. As this was time-driven rather than an event-driven analysis, the primary efficacy results may have been affected by the high rates of discontinuation while on tamoxifen monotherapy and the timing of the exemestane switch [39]. The analysis is complicated by the fact that almost $30 \%$ and $20 \%$ of patients randomized to tamoxifen and exemestane, respectively, discontinued their assigned drug before a protocolamended crossover of all participants at 2.75 years.

\section{Switch and sequence AI therapy}

\subsection{Switch study design}

In this study design, patients who have already received between 2 and 3 years of tamoxifen and have remained disease-free are randomized to either continue tamoxifen or switch to an AI. The population differs from that of an initial adjuvant trial such as ATAC or BIG 1-98, which randomizes patients at the time of surgery. Because patients have already shown a favorable response to endocrine therapy, they may be expected to have a better prognosis. In addition, by definition, the switch design excludes patients whose disease recurs early (i.e., within 2 years) while on tamoxifen, including those at risk for early DM [4]. The IES examined a switch to exemestane [14, 41], whereas the ARNO 95, ITA, and ABCSG 8 studies examined a switch to anastrozole versus continued tamoxifen [43, 55]. Combined results of smaller trials (i.e., ARNO 95/ABCSG 8/ITA) as well as the larger IES results are outlined below.

\subsection{IES: switching from tamoxifen to exemestane}

In this double-blind study, patients $(N=4,742)$ with $\mathrm{ER}+$ or ER-unknown breast cancer who tolerated 2 to 3 years of tamoxifen without relapse were randomized to either switch to exemestane or continue with tamoxifen for the remainder of 5 years $[14,41]$. The study thus excluded patients who 
relapsed during prior tamoxifen treatment and selects for patients at less risk of relapse or who are more sensitive to hormonal therapy. Since patients had ER+ or unknown disease, the ITT analysis includes patients who were later found to have ER- disease. The population differs from that of BIG 1-98, which included HR+ patients as defined by positivity for ER, PgR, or both [11, 14].

At a follow-up of 55.7 months, in the most clinically relevant ER+/unknown group, there was a significant improvement in DFS among patients who switched to exemestane $(\mathrm{HR}=0.75 ; P=0.0001)$ compared with those who remained on tamoxifen. The risk of DM (TTDR) was also significantly reduced in exemestane-treated patients in the $\mathrm{ER}+/$ unknown group $(\mathrm{HR}=0.83 ; P=0.03)$. There were 210 deaths in the exemestane group, versus 251 in the tamoxifen group (17\% relative reduction; $P=0.05)$ [14]. Following adjustment for hormone receptor status, there was a significant OS benefit in the ER+/unknown group [14]. In the ITT population, 39 deaths were prevented by switching to exemestane, with 15 fewer deaths related to breast cancer (151 exemestane vs. 166 tamoxifen) [14]. Similarly, an updated survival and safety analysis at a median of 91 months of follow-up when at least $91 \%$ of surviving patients had a minimum of 6 years follow-up available demonstrated continuing improvement in OS with 352 deaths occurring in the exemestane group compared with 405 deaths in the tamoxifen group ( $\mathrm{HR}=0.86 ; P=0.04$ ) [56].

\subsection{ABCSG 8/ARNO 95/ITA}

A combined analysis of the similarly designed ABCSG 8 and ARNO 95 trials at 28 months of follow-up reported a significant benefit in event-free survival (EFS; HR $=0.60 ; P$ $=0.0009)$ and in DM-free survival $(\mathrm{HR}=0.61 ; P=0.0067)$ for patients who switched to anastrozole after 2 years on tamoxifen relative to those who continued on tamoxifen [42]. However, there was no significant benefit in OS (97\% switch to anastrozole vs. $96 \%$ tamoxifen alone; $P=0.16$ ) [42]. Similarly, a meta-analysis of ABCSG 8, ARNO 95, and ITA showed significant improvement in DFS (HR= $0.59 ; P<0.0001)$, EFS $(\mathrm{HR}=0.55 ; P<0.0001)$, DM-free survival $(\mathrm{HR}=0.61 ; P=0.002)$, and $\mathrm{OS}(\mathrm{HR}=0.71 ; P=0.04)$ [57], although differences in trial designs and patient populations warrant a cautious interpretation of results. The individual results of ARNO 95 (for DFS, HR $=0.66 ; P$ $=0.049$ ) [55], $\mathrm{ABCSG} 8$ (for $\mathrm{EFS}, \mathrm{HR}=0.68 ; P=0.02$ ) [58], and ITA (for DFS, HR $=0.35 ; P=0.001$ ) also support a switch to anastrozole following 2 years of prior tamoxifen. Again, although these trials show a significant DFS benefit in switching patients from tamoxifen to anastrozole or exemestane, it is not possible to directly compare outcomes of upfront or switch trials because of differences in the trial designs and populations analyzed.
6.4 ABCSG 8 trial: sequential therapy with tamoxifen to anastrozole

This open-label trial examined the efficacy of initial tamoxifen therapy followed by a switch to anastrozole after 2 years compared with tamoxifen monotherapy in patients with hormone-sensitive breast cancer [42]. ABCSG 8 differed from other sequential trials in that it randomized newly diagnosed patients rather than randomizing at the point of switch [58], allowing a more appropriate analysis of all events from the time of randomization and not just from the time of the switch. Notably, when including all events from randomization at a median of 55 months, the reduction in risk of recurrence $(\mathrm{HR}=0.76 ; P=0.07)$ achieved by switching to anastrozole was not significant [59]. In an updated analysis (median 72 months) for relapse-free survival (RFS) in the sequence sample $(N=2,922)$, there was a $21 \%$ improvement with the switch to anastrozole ( $\mathrm{HR}=0.79 ; P=$ 0.038) [59]. While these data, like the IES data, support the contention that switching to an AI after 2 years is superior to continuing tamoxifen, the trial is limited by its open-label design, and the updated results are complicated by crossover of patients from tamoxifen to anastrozole $(N=82)$ and withdrawals during the tamoxifen therapy period. Furthermore, while primary endpoints for the 55-month analysis looked at EFS but not death from other causes, the 72-month analysis included both RFS and death from other causes, making a putative benefit difficult to extrapolate [59]. It is also noteworthy that although the trial population was at low to intermediate risk with ductal G1/2 and lobular tumors and no chemotherapy [59], the 10-year cumulative incidence of death in the tamoxifen arm was $20 \%$, similar to outcomes in the tamoxifen arm of other AI trials in a higher-risk patient population.

\subsection{BIG 1-98: STA}

Based on the early data, the design of BIG 1-98 was modified early during the course of enrollment to allow for five additional pairwise comparisons, with analyses commencing at randomization [16]. The design was amended to address the clinically relevant question of sequential therapy, and although not a protocol-defined analysis, given the proven superior efficacy of letrozole versus tamoxifen, two of the pairwise comparisons were considered the most clinically relevant and the focus of this analysis: sequential tamoxifen to letrozole therapy versus letrozole monotherapy $(N=$ $3,094)$, and sequential letrozole to tamoxifen therapy versus letrozole monotherapy $(N=3,086)$, with a median follow-up of 71 months. It is important to distinguish the design of the sequential therapy analysis (STA) from other trials, as it allowed for the evaluation of sequential therapy versus letrozole monotherapy in a double-blind manner, including 
the use of an AI before tamoxifen, which had not been previously investigated [16].

Results demonstrated that sequential therapy was not superior to letrozole monotherapy. DFS ( $\mathrm{HR}=1.05 ; 99 \%$ CI, 0.84-1.32), OS (HR=1.13; 99\% CI, 0.83-1.53), and TTDR (HR $=1.22 ; 99 \% \mathrm{CI}, 0.88-1.69)$ favored letrozole monotherapy over sequential therapy with tamoxifen then letrozole. TTDR ( $\mathrm{HR}=1.05$; 99\% CI, 0.75-1.47) favored letrozole over letrozole followed by tamoxifen. Thus, women who received sequential therapy in either order were at greater risk for DM than those receiving monotherapy. Because BIG 1-98 was designed as superiority trial, a lack of difference between groups cannot be inferred as equivalence between treatments.

At 2 and 5 years, there were fewer breast cancer events in the letrozole monotherapy group than in the tamoxifen to letrozole group in both $\mathrm{N}+$ patients ( 2 years: $4.7 \%$ vs. $7.9 \%$; 5 years: $12.4 \%$ vs. $14.7 \%$ ) and $\mathrm{N}-$ patients ( 2 years: $0.9 \%$ vs. $1.3 \%$; 5 years: $3.5 \%$ vs. $4.9 \%$ ). The number of breast cancer events in the letrozole monotherapy group was similar to that in the letrozole to tamoxifen group in $\mathrm{N}+$ patients (2 years: $3.9 \%$ vs. $4.7 \%$; 5 years: $12.4 \%$ vs. $12.5 \%$ ), but there were fewer breast cancer events in the letrozole monotherapy group in $\mathrm{N}-$ patients $(2$ years: $0.9 \%$ vs. $1.5 \%$; 5 years: $3.5 \%$ vs. $3.9 \%$ ) [16]. These results suggest that adjuvant endocrine therapy should begin with letrozole, unless contraindicated. In addition, patients initiated on tamoxifen may be safely switched to letrozole, if indicated (e.g., for SAEs).

\subsection{TEAM: STA}

Recently, results for the second of two co-primary endpoints of the TEAM trial comparison of sequential therapy versus exemestane monotherapy were reported [40]. At a median follow-up of 5.1 years, and with $60 \%$ of patients having completed 5 years of treatment, there was no difference in efficacy between 5 years of upfront exemestane compared with the sequence of tamoxifen to exemestane. In the ITT analysis, there was no statistical difference between the two arms in either DFS (HR $=0.97$; 95\% CI, 0.88-1.08; $P=0.604$ ), TTR (HR $=0.94 ; 95 \%$ CI, $0.83-1.06 ; P=0.293)$, or OS $(\mathrm{HR}=1.00 ; 95 \% \mathrm{CI}, 0.89$ 1.14; $P=0.999)$. The investigators concluded that for PMW with endocrine-sensitive early breast cancer, both approaches are appropriate, and further translational research in the trial population may identify patients who could benefit more from one strategy over the other.

\subsection{Effect on practice recommendations}

In contrast to the 2007 St Gallen International Expert Consensus, the 2009 panel preferred AIs as initial adjuvant therapy, particularly in higher-risk patients [17]. In addition, the IBCSG-based on updated results of BIG 1-98 demonstrating superior OS with letrozole versus tamoxifen-now recommends starting adjuvant endocrine therapy with letrozole, especially in patients at higher risk for early recurrence [60]. If required, patients can be switched to tamoxifen, but only after letrozole treatment for the first 2 years [60].

\section{Extended AI therapy}

\subsection{Extended study design}

In the extended adjuvant trial design, patients who received the full course of adjuvant tamoxifen for 5 years and showed no relapses or AEs during tamoxifen treatment were randomized to receive further treatment with an $\mathrm{AI}$ or a placebo.

\subsection{MA.17: Extended therapy with letrozole}

This double-blind, randomized, placebo-controlled trial examined the efficacy of letrozole as extended adjuvant therapy in PMW $(N=5,187)$ with HR+ or receptor status-unknown disease after completing 4.5-6 years of upfront tamoxifen [61]. At a median follow-up of 30 months, women treated with letrozole had significantly better DFS $(\mathrm{HR}=0.58 ; P=$ 0.00004 ) and distant DFS (DDFS; HR $=0.60 ; P=0.002$ ) than those treated with placebo. Although OS was not significantly improved in the ITT population ( $\mathrm{HR}=0.82 ; P=0.3$ ), OS was significantly better with letrozole in $\mathrm{N}+$ patients (HR $=0.61 ; P=0.04)$ [44]. The highly significant DFS, DDFS, and OS benefit led to unblinding of the trial approximately 1 year earlier than planned, when $66 \%$ of patients receiving placebo chose to switch to letrozole, while $34 \%$ elected no further treatment [62]. After a median follow-up of 5.3 years, women who chose to switch to letrozole demonstrated significant improvements in DFS $(\mathrm{HR}=0.37 ; P<0.0001)$ and DDFS $(\mathrm{HR}=0.39 ; P=0.004)$ compared with patients who chose to terminate treatment [62]. Furthermore, examination of the outcomes of the trial indicated that letrozole improves DFS and DDFS (61\% reduction in risk of metastasis) even when there has been a substantial period of time (median, 2.8 years; range 1.17.1 years) since the discontinuation of prior adjuvant therapy [62]. These findings demonstrate that women who have completed 5 years of adjuvant tamoxifen can benefit from continuing treatment with letrozole, even if some time has elapsed since discontinuing tamoxifen.

Interestingly, recent data on a subset of women in MA.17 $(n=889)$ who were premenopausal when initially diagnosed demonstrated that extended adjuvant therapy with letrozole is significantly more effective in improving DFS in women 
who are premenopausal at the time of the primary diagnosis $(P=0.02)$ [63]. The benefit of letrozole was significantly greater in premenopausal women $(\mathrm{HR}=0.25 ; 95 \% \mathrm{CI}, 0.12-$ $0.51)$ than in postmenopausal women $(\mathrm{HR}=0.69 ; 95 \% \mathrm{CI}$, $0.52-0.91)$. Women who are premenopausal at the time of diagnosis but become postmenopausal anytime before or during adjuvant tamoxifen therapy should therefore be considered for extended adjuvant therapy with letrozole.

\subsection{ABCSG 6a: extended therapy with anastrozole}

This open-label, non-controlled trial examined extended adjuvant therapy in $856 \mathrm{HR}+$ PMW. Patients who completed either 5 years of tamoxifen, or tamoxifen plus aminoglutethimide for 2 years followed by tamoxifen monotherapy for 3 years, were randomized to either an additional 3 years of anastrozole or no further treatment [45]. At a median followup of 62.3 months, the risk of recurrence (local, contralateral, or distant) was significantly reduced $(\mathrm{HR}=0.62 ; P=0.031)$, as was the risk of $\mathrm{DM}(\mathrm{HR}=0.53 ; P=0.034)$, in patients treated with anastrozole compared with those receiving no treatment, but no benefit was observed in OS (HR=0.89; $P=$ 0.57) [45]. Although limited by its open-label design, the results support the use of an $\mathrm{AI}$ in patients with a full course of prior tamoxifen treatment.

\subsection{NSABP B-33: extended therapy with exemestane}

In this trial, ER+ PMW completing 5 years of tamoxifen were randomized to receive exemestane or placebo for a further 5 years [47]. When results from the MA.17 trial showed that extended adjuvant therapy with letrozole after 5 years of tamoxifen provided significant benefits, the B-33 trial was closed prematurely, so that exemestane therapy could be offered to patients receiving placebo. At the time of unblinding, 1,598 patients had been randomly assigned; $72 \%$ in the exemestane group continued on exemestane, and $44 \%$ in the placebo group elected to receive exemestane. As a result, the trial failed to reach its target accrual, and the primary endpoint of the trial (DFS) did not reach significance after a median follow-up of 30 months (relative risk $(\mathrm{RR})=0.68 ; P=0.07)$ [47]. A statistically significant improvement was only shown in the 4-year analysis RFS $(96 \%$ vs. $94 \%$; $\mathrm{RR}=0.44 ; P=004)$. $\mathrm{PMW}$ with $\mathrm{HR}+\mathrm{EBC}$ continue to be at risk of relapse and mortality after 5 years on tamoxifen [64].

\section{Meta-analysis of breast cancer outcomes in adjuvant trials of AIs versus tamoxifen}

The Aromatase Inhibitor Overview Group (AIOG) was founded in December 2003 by the principal investigators of all major AI clinical trials. Working in collaboration with the EBCTCG, the AIOG sought to analyze the results of almost 40,000 patients participating in AI trials of different treatment strategies.

\subsection{Upfront and switch therapy trials}

A meta-analysis of randomized trials of monotherapy and switching strategies presented during the 2008 San Antonio Breast Cancer Symposium (SABCS) [65] was recently published by the AIOG [66]. Data from randomized clinical trials comparing AIs with tamoxifen either as initial monotherapy (cohort 1: ATAC and BIG 1-98/ IBCSG 18-98 trials) or after 2 to 3 years of tamoxifen (cohort 2: German Adjuvant Breast Cancer Group/ARNO, IES/BIG 2-97, ITA, and ABCSG 8 trials) were submitted to the EBCTCG and used in separate meta-analyses of the two cohorts. Primary analyses involved only PMW with ER+ tumors. Cohort 1 included 9,856 patients with a mean follow-up of 5.8 years. At 5 years, AI therapy was associated with an absolute $2.9 \%$ (standard error (SE) = $0.7 \%)$ decrease in recurrence $(9.6 \%$ for $\mathrm{AI}$ vs. $12.6 \%$ for tamoxifen; $2 P<0.00001)$ and a nonsignificant absolute $1.1 \%(\mathrm{SE}=0.5 \%)$ decrease in breast cancer mortality ( $4.8 \%$ for AI vs. $5.9 \%$ for tamoxifen; $2 P=0.1$ ). Cohort 2 included 9,015 patients with a mean follow-up of 3.9 years. At 3 years from switching treatment (approximately 5 years after starting hormonal treatment), AI therapy was associated with an absolute $3.1 \%(\mathrm{SE}=0.6 \%)$ decrease in recurrence $(5.0 \%$ for $\mathrm{AI}$ vs. $8.1 \%$ for tamoxifen since switch; $2 P<0.00001)$ and an absolute $0.7 \%(\mathrm{SE}=0.3 \%)$ decrease in breast cancer mortality (1.7\% for AI vs. $2.4 \%$ for tamoxifen since switch; $2 P=$ 0.02). There was no apparent heterogeneity in the proportional recurrence reduction with respect to age, nodal status, tumor grade, or $\mathrm{PgR}$, status and no indication of an increase in non-breast cancer deaths with AIs in either cohort. The absence of a statistically significant difference in non-breast cancer death and overall mortality in both cohorts was considered to be a possible indication in favor of the overall safety of treatment with AIs. It was concluded that AI-treated patients have statistically significant improvements in RFS in both cohorts, and AIs provide a significantly lower recurrence rate in both upfront and switching strategies compared with tamoxifen ( $23 \%$ and $40 \%$, respectively). An OS benefit was only seen in cohort 2 (patients who switched to AIs). The lack of a significant difference in breast cancer mortality in cohort 1 is probably time-dependent, and further followup may be needed, given the experience with tamoxifen. It is noteworthy that the above data cannot answer the question whether the switching approach is better than the upfront approach. 


\subsection{Extended therapy trials}

A meta-analysis of extended AI therapy trials was presented by the AIOG during the 2009 SABCS [67]. Included were efficacy results from the MA.17, ABCSG 6a, NSABP B-33, and Adjuvant post-Tamoxifen Exemestane versus Nothing Applied (ATENA) trials. The ATENA trial was closed prematurely - after only 448 of the planned 1,803 patients had been enrolled - because of results of the MA.17 trial [68]. Taken together, the extended AI therapy trials suggest that continuing treatment with an AI after 5 years of tamoxifen is better than no further treatment. However, this has only been proven conclusively with letrozole [44-46, 62 , 67]. The optimal duration of extended adjuvant therapy remains undetermined.

\section{Discussion}

While the risk of recurrence may be greater for patients with $\mathrm{N}+$ status, all patients with $\mathrm{EBC}$ are at risk for recurrence, including those with $\mathrm{N}-$ tumors of high grade or large size [4, 19]. Prognostic factors such as high Ki67 expression may also identify a subgroup of patients (including those with $\mathrm{N}-$ disease) at high risk for relapse and poor outcome [20]. Preventing DM is critical to improving outcomes; they represent the majority of EBC recurrence events, which peak approximately 2 years after surgery [4], and are associated with the poorest prognosis compared with locoregional and contralateral recurrences $[5,8,10]$. Therapies that effectively reduce the risk of early DM are, therefore, likely to improve long-term outcomes, including OS.

Major clinical trials clearly demonstrate a benefit of AI therapy over tamoxifen in multiple adjuvant treatment settings. In the initial adjuvant setting, both anastrozole and letrozole have proven superior to tamoxifen in improving DFS, whereas results for exemestane in this setting remain inconclusive. In addition, initial adjuvant letrozole has been shown to significantly reduce early DM $[6,11]$. This was also shown with anastrozole, but to a significantly lesser extent $[3,48]$. The significant reduction in the incidence of early DM may in part explain the emergent OS benefit with letrozole over tamoxifen with long-term follow-up [16, 49], whereas no improvement in survival has been seen for anastrozole over tamoxifen with long-term follow-up [15]. Letrozole appears to be more potent than anastrozole in terms of inhibiting estrogen [37, 69]. However, whether this results in differences in clinical efficacy, remains to be determined in the directly comparative FACE trial.

Adjuvant AI trials including the IES, ABCSG 8, and ARNO 95 show that switching to an AI (anastrozole or exemestane) after 2 to 3 years of tamoxifen is better than continuing tamoxifen. However, it is not possible to compare results from these switch trials with upfront trials, because the switch trials included only patients who were disease-free at the time of randomization. Recently reported results from the STA of BIG 1-98 further suggest that using a sequence of tamoxifen and letrozole, in either order, is not superior to using letrozole monotherapy upfront [16], which has already been shown to be better than tamoxifen monotherapy. Exemestane was not shown to be better than sequential therapy with tamoxifen and exemestane, but exemestane also was not shown to be better than tamoxifen in the upfront setting during the first 2.75 crucial years of treatment [40]. Lastly, extended adjuvant trials have demonstrated that continuing adjuvant endocrine therapy with an AI improves outcomes when compared with no further treatment in patients who have received the full course of prior tamoxifen, although this benefit has been conclusively shown only with letrozole.

\section{Conclusions}

Recent clinical trials have demonstrated the superiority of initial treatment with AIs instead of tamoxifen, with letrozole providing the most reduction in DM (30\%) and having the greatest impact on reducing the risk of early DM $[6,11]$. As reported by the updated analyses of BIG 1-98, this is translating into an OS benefit with longer follow-up $[16,49,51]$. A statistically significant effect of anastrozole on reducing DM was observed only after a longer followup (i.e., after 100 months), well beyond the crucial period during which patients are at the highest risk of DM [15]. The STA of BIG 1-98 has shown that sequential therapy with tamoxifen and letrozole, in either order, is not superior to initial adjuvant letrozole [16], and recently published St. Gallen guidelines seem to recommend the use of an AI upfront, particularly in patients at higher risk of early relapse [17]. However, for patients unable to begin adjuvant endocrine therapy with an AI, switch and extended adjuvant trials demonstrate that at the least, adding an AI at some point after tamoxifen can help reduce the risk of recurrence and improve outcome. While efforts to stratify patients into prognostic subgroups are ongoing, it remains difficult to quantify the risk of recurrence for an individual patient. Until risk assessment strategies are more conclusively validated in clinical trials, all patients should be considered candidates for upfront AI treatment to effectively minimize early DM and improve survival.

Acknowledgments Financial support for medical editorial assistance was provided by Novartis Pharmaceuticals. We thank Maria Soushko, PhD, of Phase Five Communications Inc. for medical editorial assistance with this manuscript. 
Open Access This article is distributed under the terms of the Creative Commons Attribution Noncommercial License which permits any noncommercial use, distribution, and reproduction in any medium, provided the original author(s) and source are credited.

\section{References}

1. American Cancer Society (2009). Cancer Facts \& Figures 2009. Atlanta: American Cancer Society.

2. Saphner, T., Tormey, D. C., \& Gray, R. (1996). Annual hazard rates of recurrence for breast cancer after primary therapy. Journal of Clinical Oncology, 14(10), 2738-2746.

3. Houghton, J. (2006). Initial adjuvant therapy with anastrozole (A) reduces rates of early breast cancer recurrence and adverse events compared with tamoxifen (T) - data reported on behalf of the ATAC ('Arimidex', tamoxifen, alone or in Combination) Trialists' group. Annals of Oncology, 17(Suppl. 9), 94. Abstract 243PD.

4. Mansell, J., Monypenny, I. J., Skene, A. I., Abram, P., Carpenter, R., Gattuso, J. M., et al. (2009). Patterns and predictors of early recurrence in postmenopausal women with estrogen receptorpositive early breast cancer. Breast Cancer Research and Treatment, 117(1), 91-98.

5. Lamerato, L., Havstad, S., Gandhi, S., Jones, D., \& Chlebowski, R. (2005). Breast cancer recurrence and related mortality in US patients with early breast cancer. Journal of Clinical Oncology, 23 (16S), 62s. Abstract 738.

6. Mauriac, L., Keshaviah, A., Debled, M., Mouridsen, H., Forbes, J. F., Thürlimann, B., et al. (2007). Predictors of early relapse in postmenopausal women with hormone receptor-positive breast cancer in the BIG 1-98 trial. Annals of Oncology, 18(5), 859-867.

7. Doughty, J. C. (2008). A review of the BIG results: the Breast International Group 1-98 trial analyses. Breast, 17(Suppl 1), S9-S14.

8. Doughty, J., Mansell, J., Monypenny, I., Skene A., Abram P., Carpenter R., et al. (2009) Distant recurrence and subsequent mortality in breast cancer patients treated at 5 UK centres. Presented at the St. Gallen Breast Cancer Conference; March 11-14, 2009; St. Gallen, Switzerland. Abstract 188.

9. Glas, A., Floore, A., Delahaye, L. J., Witteveen, A. T., Pover, R. C., Bakx, N., et al. (2006). Converting a breast cancer microarray signature into a high-throughput diagnostic test. BMC Genomics, 7, 278.

10. Lê, M. G., Arriagada, R., Spielmann, M., Guinebretiére, J.-M., \& Rochard, F. (2002). Prognostic factors for death after an isolated local recurrence in patients with early-stage breast carcinoma. Cancer, 94(11), 2813-2820.

11. Thurlimann, B., Keshaviah, A., Coates, A. S., Mouridsen, H., Mauriac, L., Forbes, J. F., et al. (2005). A comparison of letrozole and tamoxifen in postmenopausal women with early breast cancer. The New England Journal of Medicine, 353(26), 2747-2757.

12. Howell, A., Cuzick, J., Baum, M., Buzdar, A., Dowsett, M., Forbes, J. F., et al. (2005). Results of the ATAC (Arimidex, Tamoxifen, Alone or in Combination) trial after completion of 5 years' adjuvant treatment for breast cancer. Lancet, 365(9453), 60-62.

13. Coates, A. S., Keshaviah, A., Thurlimann, B., Mouridsen, H., Mauriac, L., Forbes, J. F., et al. (2007). Five years of letrozole compared with tamoxifen as initial adjuvant therapy for postmenopausal women with endocrine-responsive early breast cancer: update of study BIG 1-98. Journal of Clinical Oncology, 25(5), 486-492.

14. Coombes, R. C., Kilburn, L. S., Snowdon, C. F., Paridaens, R., Coleman, R. E., Jones, S. E., et al. (2007). Survival and safety of exemestane versus tamoxifen after 2-3 years' tamoxifen treatment (Intergroup Exemestane Study): a randomised controlled trial. Lancet, 369(9561), 559-570.

15. Arimidex, Tamoxifen, Alone or in Combination (ATAC) Trialists' Group, Forbes, J. F., Cuzick, J., Buzdar, A., et al. (2008). Effect of anastrozole and tamoxifen as adjuvant treatment for early-stage breast cancer: 100-month analysis of the ATAC trial. Lancet Oncology, 9(1), 45-53.

16. Mouridsen, H., Giobbie-Hurder, A., Goldhirsch, A., Thürlimann, B., Paridaens, R., Smith, I., et al. (2009). Letrozole therapy alone or in sequence with tamoxifen in women with breast cancer. The New England Journal of Medicine, 361((8)), 766-776.

17. Goldhirsch, A., Ingle, J. N., Gelber, R. D., Coates, A. S., Thürlimann, B., Senn, H. J., et al. (2009). Thresholds for therapies: highlights of the St Gallen International Expert Consensus on the primary therapy of early breast cancer 2009 . Annals of Oncology, 20(8), 1319-1329.

18. Debled, M., MacGrogan, G., Brouste, V., Mathoulin-Pelissier, S., Durand, M., \& Mauriac, L. (2007). Prognostic factors of early distant recurrence in hormone receptor-positive, postmenopausal breast cancer patients receiving adjuvant tamoxifen therapy: results of a retrospective analysis. Cancer, 109(11), 2197-2204.

19. Goldhirsch, A., Wood, W. C., Gelber, R. D., Coates, A. S., Thurlimann, B., Senn, H. J., et al. (2007). Progress and promise: highlights of the international expert consensus on the primary therapy of early breast cancer 2007. Annals of Oncology, 18(7), 1133-1144. Erratum in: Annals of Oncology 2007; 18(11), 1917.

20. Jung, S. Y., Han, W., Lee, J. W., Ko, E., Kim, E., Yu, J. H., et al. (2009). Ki-67 expression gives additional prognostic information on St. Gallen, 2007 and Adjuvant! Online risk categories in early breast cancer. Annals of Surgical Oncology, 16(5), 1112-1121.

21. Sotiriou, C., \& Pusztai, L. (2009). Gene-expression signatures in breast cancer. The New England Journal of Medicine, 360(8), 790-800.

22. Paik, S., Shak, S., Tang, G., Kim, C., Baker, J., Cronin, M., et al. (2004). A multigene assay to predict recurrence of tamoxifentreated, node-negative breast cancer. The New England Journal of Medicine, 351(27), 2817.

23. Paik, S., Kim, C., Baker, J., Kim, W., Cronin, M., Baehner, F. L., et al. (2006). Gene expression and benefit of chemotherapy in women with node-negative, estrogen receptor- positive breast cancer. Journal of Clinical Oncology, 24(23), 3726-3734.

24. Sparano, J. A. (2006). TAILORx: trial assigning individualized options for treatment (Rx). Clinical Breast Cancer, 7(4), 347-350.

25. Dowsett, M., Cuzick, J., Wales, C., Forbes, J., Mallon, I., Salter, J., et al. (2009). Risk of distant recurrence using oncotype DX in postmenopausal primary breast cancer patients treated with anastrozole or tamoxifen: a TransATAC study. Cancer Research, 69(Suppl), 75s. Abstract 53.

26. Cardoso, F., Piccart-Gebhart, M., Van't Veer, L., Rutgers, E., \& TRANSBIG Consortium (2007). The MINDACT trial: the first prospective clinical validation of a genomic tool. Molecular Oncology, 1(3), 246-251.

27. Early Breast Cancer Trialists' Collaborative Group (EBCTCG) (2005). Effects of chemotherapy and hormonal therapy for early breast cancer on recurrence and 15-year survival: an overview of the randomised trials. Lancet, 365(9472), 1687-1717.

28. Del Mastro, L., Clavarezza, M., \& Venturini, M. (2007). Reducing the risk of distant metastases in breast cancer patients: role of aromatase inhibitors. Cancer Treat Reviews, 33(8), 681-687.

29. Fisher, B., Dignam, J., Bryant, J., \& Wolmark, N. (2001). Five versus more than five years of tamoxifen for lymph nodenegative breast cancer: updated findings from the National Surgical Adjuvant Breast and Bowel Project B-14 randomized trial. Journal of the National Cancer Institute, 93(9), 684-690.

30. Rutqvist, L. E., Johansson, H., \& Stockholm Breast Cancer Study Group (2007). Long-term follow-up of the randomized Stockholm trial on adjuvant tamoxifen among postmenopausal patients with early stage breast cancer. Acta Oncology, 46(2), 133-145.

31. Goetz, M. P., Knox, S. K., Suman, V. J., Rae, J. M., Safgren, S. L., Ames, M. M., et al. (2007). The impact of cytochrome P450 2D6 
metabolism in women receiving adjuvant tamoxifen. Breast Cancer Research and Treatment, 101(1), 113-121.

32. Schroth, W., Goetz, M. P., Hamann, U., Fasching, P. A., Schmidt, M., Winter, S., et al. (2009). Association between CYP2D6 polymorphisms and outcomes among women with early stage breast cancer treated with tamoxifen. Journal of the American Medical Association, 302(13), 1429-1436.

33. Aubert, R. E., Stanek, E. J., Yao, J., Teagarden, J. R., Subar, M., Epstein, R. S., et al. (2009) Risk of breast cancer recurrence in women initiating tamoxifen with CYP2D6 inhibitors. Journal of Clinical Oncology 27(18 S). Abstract CRA508.

34. Perez, E. A. (2007). Safety profiles of tamoxifen and the aromatase inhibitors in adjuvant therapy of hormone-responsive early breast cancer. Annals of Oncology, 18(Suppl 8), 26-35.

35. Lønning, P. E., \& Geisler, J. (2008). Aromatase inhibitors: Assessment of biochemical efficacy measured by total body aromatase inhibition and tissue estrogen suppression. The Journal of Steroid Biochemistry and Molecular Biology, 108(3-5), 196202.

36. Buzdar, A., Chlebowski, R., Cuzick, J., Duffy, S., Forbes, J., Jonat, W., et al. (2006). Defining the role of aromatase inhibitors in the adjuvant endocrine treatment of early breast cancer. Current Medical Research and Opinion, 22(8), 1575-1585.

37. Geisler, J., Helle, H., Ekse, D., Duong, N. K., Evans, D. B., Nordbø, Y., et al. (2008). Letrozole is superior to anastrozole suppressing breast cancer tissue and plasma estrogen levels. Clinical Cancer Research, 14(19), 6330-6335.

38. Baum, M., Budzar, A. U., Cuzick, J., Forbes, J., Houghton, J. H., Klijn, J. G., et al. (2002). Anastrozole alone or in combination with tamoxifen versus tamoxifen alone for adjuvant treatment of postmenopausal women with early breast cancer: first results of the ATAC randomised trial. Lancet, 359(9324), 2131-2139.

39. Jones, S. E., Seynaeve, C., Hasenburg, A., Rae, D., Vannetzel, J.-M., Paridaens, R., et al. (2009). Results of the first planned analysis of the TEAM (tamoxifen exemestane adjuvant multinational) prospective randomized phase III trial in hormone sensitive postmenopausal early breast cancer. Cancer Research, 69(Suppl), $66 \mathrm{~s}$.

40. Rea, D., Hasenburg, A., Seynaeve, C., Jones, S. E., Vannetzel, J. M., Paridaens, R., et al. (2009). Five years of exemestane as initial therapy compared to tamoxifen followed by exemestane for five years: the TEAM trial, a prospective, randomized, phase iii trial in postmenopausal women with hormone-sensitive early breast cancer. Cancer Research, 69(Suppl), s24.

41. Coombes, R. C., Hall, E., Gibson, L. J., Paridaens, R., Jassem, J., Delozier, T., et al. (2004). A randomized trial of exemestane after two to three years of tamoxifen therapy in postmenopausal women with primary breast cancer. The New England Journal of Medicine, 350(11), 1081-1092.

42. Jakesz, R., Jonat, W., Gnant, M., Mittlboeck, M., Greil, R., Tausch, C., et al. (2005). Switching of postmenopausal women with endocrine-responsive early breast cancer to anastrozole after 2 years' adjuvant tamoxifen: combined results of ABCSG trial 8 and ARNO 95 trial. Lancet, 366(9484), 455-462.

43. Boccardo, F., Rubagotti, A., Puntoni, M., Guglielmini, P., Amoroso, D., Fini, A., et al. (2005). Switching to anastrozole versus continued tamoxifen treatment of early breast cancer: preliminary results of the Italian Tamoxifen Anastrozole trial. Journal of Clinical Oncology, 23(22), 5138-5147.

44. Goss, P. E., Ingle, J. N., Martino, S., Robert, N. J., Muss, H. B., Piccart, M. J., et al. (2005). Randomized trial of letrozole following tamoxifen as extended adjuvant therapy in receptorpositive breast cancer: updated findings from NCIC CTG MA.17. Journal of the National Cancer Institute, 97(17), 1262-1271.

45. Jakesz, R., Greil, R., Gnant, M., Schmid, M., Kwasny, W., Kubista, E., et al. (2007). Extended adjuvant therapy with anastrozole among postmenopausal breast cancer patients: results from the randomized Austrian Breast and Colorectal Cancer Study Group Trial 6a. Journal of the National Cancer Institute, 99(24), 1845-1853. Erratum in: Journal of the National Cancer Institute 2008; 100(3), 226.

46. Mamounas, E. P. (2001). Adjuvant exemestane therapy after 5 years of tamoxifen: rationale for the NSABP B-33 trial. Oncology, 15(5 Suppl 7), 35-39.

47. Mamounas, E. P., Jeong, J. H., Wickerham, D. L., Smith, R. E., Ganz, P. A., Land, S. R., et al. (2008). Benefit from exemestane as extended adjuvant therapy after 5 years of adjuvant tamoxifen: intention-to-treat analysis of the National Surgical Adjuvant Breast and Bowel Project B-33 trial. Journal of Clinical Oncology, 26(12), 1965-19671.

48. Howell, A., Forbes, J., \& Cuzick, J. (2009) Initial adjuvant therapy with anastrozole - early and late event data from the arimidex, tamoxifen, alone or in combination (ATAC) trial in the hormone-responsive population. Presented at the St. Gallen Breast Cancer Conference; March 11-14; St. Gallen, Switzerland. Abstract 0130

49. Thurlimann B; BIG 1-98 Collaborative Group (2009) Letrozole vs. tamoxifen as adjuvant endocrine therapy for postmenopausal women with receptor positive breast cancer. Update of the BIG 1-98 primary core analysis. Presented at the St. Gallen Breast Cancer Conference; March 11-14; St. Gallen, Switzerland. Abstract 0161.

50. Robins, J. M., \& Finkelstein, D. M. (2000). Correcting for noncompliance and dependent censoring in an AIDS Clinical Trial with inverse probability of censoring weighted (IPCW) log-rank tests. Biometrics, 56(3), 779-788.

51. Regan, M. M., Colleoni, M., Giobbie-Hurder, A., Thuerlimann, B., Mouridsen, H., Mauriac, L., et al. (2009) Adjusting for selective crossover in analyses of letrozole (Let) versus tamoxifen (Tam) in the BIG 1-98 trial. Cancer Research 69(24 Suppl). Abstract 16.

52. DeBoer, R., Burris, H., Monnier, A., Mouridsen, H., O'Shaughnessy, J., \& McIntyre, K. (2006). The head to head trial: letrozole vs. anastrozole as adjuvant treatment of postmenopausal patients with node positive breast cancer. Journal of Clinical Oncology, 24(18S), 582s. Abstract 10672.

53. Monnier, A. (2006). Refining the postmenopausal breast cancer treatment paradigm: the FACE trial. Expert Review of Anticancer Therapy, 6(10), 1355-1359.

54. van de Velde, C., Seynaeve, C. H., Hasenburg, A., Rea, D., Vannetzel, J. M., Paridaens, R., et al. (2009). Results of the TEAM (tamoxifen exemestane adjuvant multinational) prospective randomized phase III trial in hormone sensitive postmenopausal early breast cancer. European Journal of Cancer Supplements, 7(3), 1. Abstract 2BA.

55. Kaufmann, M., Jonat, W., Hilfrich, J., Eidtmann, H., Gademann, G., Zuna, I., et al. (2007). Improved overall survival in postmenopausal women with early breast cancer after anastrozole initiated after treatment with tamoxifen compared with continued tamoxifen: the ARNO 95 Study. Journal of Clinical Oncology, 25 (19), 2664-2670.

56. Bliss, J. M., Kilburn, L. S., Coleman, R. E., Forbes, J. F., Coates, A. S., Jones, S. E., et al. (2009) Disease related outcome with long term follow-up: an updated analysis of the Intergroup Exemestane Study (IES). Cancer Research 69(24 Suppl). Abstract 12.

57. Jonat, W., Gnant, M., Boccardo, F., Kaufmann, M., Rubagotti, A., Zuna, I., et al. (2006). Effectiveness of switching from adjuvant tamoxifen to anastrozole in postmenopausal women with hormone-sensitive early-stage breast cancer: a meta-analysis. The Lancet Oncology, 7(12), 991-996.

58. Jakesz, R., Gnant, N., Greil, R., Tausch, C., Samonigg, H., Kwasny, W., et al. (2005). The benefits of sequencing adjuvant tamoxifen and anastrozole in postmenopausal women with hormone-responsive early breast cancer: 5-year analysis of 
ABCSG Trial 8. Breast Cancer Research and Treatment, 94(Suppl 1), S10. Abstract 13

59. Jakesz, R., Gnant, M., Griel, R., Tausch, C., Samonigg, H., Kwasny, W., et al. (2009). Tamoxifen and anastrozole as a sequencing strategy in postmenopausal women with hormoneresponsive early breast cancer: updated data from the Austrian breast and colorectal cancer study group trial 8. Cancer Research, 69(Suppl), 67s. Abstract 14.

60. Mouridsen, H. T., Giobbie-Hurder, A., Mauriac, L., Paridaens, R., Colleoni, M., Thuerlimann, B., et al. (2009). BIG 1-98: A randomized double-blind phase III study evaluating letrozole and tamoxifen given in sequence as adjuvant endocrine therapy for postmenopausal women with receptor-positive breast cancer. Cancer Research, 69(Suppl 2), 66s. Abstract 13.

61. Goss, P. E., Ingle, J. N., Martino, S., Robert, N. J., Muss, H. B., Piccart, M. J., et al. (2003). A randomized trial of letrozole in postmenopausal women after five years of tamoxifen therapy for early-stage breast cancer. The New England Journal of Medicine, 349(19), 1793-1802.

62. Goss, P. E., Ingle, J. N., Pater, J. L., Martino, S., Robert, N. J., Muss, H. B., et al. (2008). Late extended adjuvant treatment with letrozole improves outcome in women with early-stage breast cancer who complete 5 years of tamoxifen. Journal of Clinical Oncology, 26(12), 1948-1955.

63. Goss, P. E., Ingle, J. N., Martino, S., Robert, N., Muss, H., Shepherd, L., et al. (2009) Outcomes of Women Who Were Premenopausal at Diagnosis of Early Stage Breast Cancer in the NCIC CTG MA17 Trial. Cancer Research 69(24 Suppl):Abstract 13.

64. Kennecke, H. F., Olivotto, I. A., Speers, C., Norris, B., Chia, S. K., Bryce, C., et al. (2007). Late risk of relapse and mortality among postmenopausal women with estrogen responsive early breast cancer after 5 years of tamoxifen. Annals of Oncology, 18 (1), 45-51.

65. Ingle, J. N., Dowsett, M., Cuzick, J., Davies, C., \& For the Aromatase Inhibitors Overview Group (AIOG). (2009). Aromatase inhibitors versus tamoxifen as adjuvant therapy for postmenopausal women with estrogen receptor positive breast cancer: meta-analyses of randomized trials of monotherapy and switching strategies. Cancer Research, 69(Suppl 2), 66s. Abstract 12.

66. Dowsett, M., Cuzick, J., Ingle, J., Coates, A., Forbes, J., Bliss, J., et al. (2010). Meta-Analysis of Breast Cancer Outcomes in Adjuvant Trials of Aromatase Inhibitors Versus Tamoxifen. Journal of Clinical Oncology, 28(3), 509-518.

67. Goss, P. E., Mamounas, E. P., Jakesz, R., Markopoulos, C., Dowsett, M., Peto, R., et al. (2009) Aromatase inhibitors (AIs) versus not (placebo/observation) as late extended adjuvant therapy for postmenopausal women with early stage breast cancer (BC): overviews of randomized trials of AIs after $\sim 5$ years of tamoxifen. Cancer Research 69(24 Suppl), Abstract 4081.

68. Markopoulos, C., Dafni, U., Misitzis, J., Zobolas, V., Tzoracoleftherakis, E., Koukouras, D., et al. (2009). Extended adjuvant hormonal therapy with exemestane has no detrimental effect on the lipid profile of postmenopausal breast cancer patients: final results of the ATENA lipid substudy. Breast Cancer Research, 11(3), R35.

69. Dixon, J. M., Renshaw, L., Young, O., Murray, J., Macaskill, E. J., McHugh, M., et al. (2008). Letrozole suppresses plasma estradiol and estrone sulphate more completely than anastrozole in postmenopausal women with breast cancer. Journal of Clinical Oncology, 26(10), 1671-1676. 